\title{
Right hemispheric contributions to fine auditory temporal discriminations: high-density electrical mapping of the duration mismatch negativity (MMN)
}

\author{
Pierfilippo De Sanctis ${ }^{1,2}$, Sophie Molholm ${ }^{1,2}$, Marina Shpaner ${ }^{1,2}$, Walter Ritter ${ }^{1,2}$ and John J. Foxe $e^{1,2 *}$ \\ The Cognitive Neurophysiology Laboratory, Nathan S. Kline Institute for Psychiatric Research, Program in Cognitive Neuroscience and Schizophrenia, \\ Orangeburg, NY, USA \\ 2 Program in Cognitive Neuroscience, Departments of Psychology and Biology, City College of the City University of New York, New York, USA
}

\section{Edited by:}

Barry E. Stein, Wake Forest University, USA

\section{Reviewed by:}

Gregg H. Recanzone, University of California, USA

Mark Wallace, Vanderbilt University, USA

\section{${ }^{*}$ Correspondence:}

John J. Foxe, Program in Cognitive Neuroscience, Departments of

Psychology and Biology, City College of the City University of New York, 138th

Street and Convent Avenue, New York, New York 10031, USA.

e-mail: foxe@nki.rfmh.org
That language processing is primarily a function of the left hemisphere has led to the supposition that auditory temporal discrimination is particularly well-tuned in the left hemisphere, since speech discrimination is thought to rely heavily on the registration of temporal transitions. However, physiological data have not consistently supported this view. Rather, functional imaging studies often show equally strong, if not stronger, contributions from the right hemisphere during temporal processing tasks, suggesting a more complex underlying neural substrate. The mismatch negativity (MMN) component of the human auditory evoked-potential provides a sensitive metric of duration processing in human auditory cortex and lateralization of $\mathrm{MMN}$ can be readily assayed when sufficiently dense electrode arrays are employed. Here, the sensitivity of the left and right auditory cortex for temporal processing was measured by recording the MMN to small duration deviants presented to either the left or right ear. We found that duration deviants differing by just 15\% (i.e. rare 115 ms tones presented in a stream of 100 ms tones) elicited a significant $\mathrm{MMN}$ for tones presented to the left ear (biasing the right hemisphere). However, deviants presented to the right ear elicited no detectable MMN for this separation. Further, participants detected significantly more duration deviants and committed fewer false alarms for tones presented to the left ear during a subsequent psychophysical testing session. In contrast to the prevalent model, these results point to equivalent if not greater right hemisphere contributions to temporal processing of small duration changes.

Keywords: hemispheric asymmetry, auditory temporal resolution, event-related potentials

\section{INTRODUCTION}

Cerebral dominance is a key organizational feature of the brains of Homo Sapiens with some arguing that it may even be a uniquely human trait (Crow, 2006), although evidence seems solid that it is also present in our nearest primate relatives (e.g. Gannon et al., 1998). Perhaps the most researched aspect of lateralization is the left hemisphere ( $\mathrm{LH}$ ) dominance for language, but many other perceptual and cognitive functions also show hemispheric asymmetries, such as the right hemisphere $(\mathrm{RH})$ specialization for visuo-spatial attention (e.g. Foxe et al., 2003; Heilman and Van Den Abell, 1980). The fact that language is a left hemisphere dominant function has led some to hypothesize that auditory temporal discrimination will be particularly well tuned in the left hemisphere, since speech discrimination relies so heavily on the registration of rapid temporal transitions and the discrimination of short temporal differences in the timing of segments (see e.g. Brown and Nicholls, 1997; Brown et al., 1999; Fitch et al., 1993; Leek and Brandt, 1983; Shannon et al., 1995). For example, a difference of just $20 \mathrm{~ms}$ in "voice-onset time," the time between when the vocal chords begin to vibrate and the mouth opens to release the sound, can change the perception of a phoneme from /ba/ to /pa/ (Abramson, 1977). Behavioral results seem to support this notion. That is, using gap-detection tasks or sequence-judgment tasks, many researchers have found that performance is best when such stimuli are presented to the right ear (i.e. biasing left hemisphere involvement; e.g. Lackner and Teuber, 1973; Sulakhe et al., 2003; Vroon et al., 1977). A comprehensive review by Nicholls (1996) found that more than two-thirds of these types of behavioral studies implicated the left hemisphere.

However, physiological data using both hemodynamic imaging and electrophysiological measures present a somewhat more complex scenario. Recent fMRI studies actually favor greater involvement of the right hemisphere in duration processing than one might have expected based on the earlier behavioral work. For example, Reiterer et al. (2005) presented tones ranging from 100 to $400 \mathrm{~ms}$ in duration, which had to be compared to a reference tone of $200 \mathrm{~ms}$, resulting in duration differences from 0 to $200 \mathrm{~ms}$. They found significantly increased activity in the right hemisphere, including the superior temporal and middle temporal gyri, that correlated with better performance in this two-pair duration discrimination task. Similarly, Harrington et al. (2004) found a correlation between fMRI activity and duration discrimination accuracy in areas that were exclusively located in the right hemisphere. In both studies, performance level was accompanied by a corresponding increase in hemodynamic activity that was solely observed in the right hemisphere. However, since fMRI measures of hemodynamics have very low temporal resolution, it remains 
unclear if the performance-related modulation of the fMRI signal reflects early sensory-perceptual encoding of temporal information or higher-order processing, e.g. decision making.

Results based on clinical studies are also rather puzzling. von Steinbüchel et al. (1999) compared patients with focal left or right hemispheric lesions to control subjects, showing that only patients with a lesion in the $\mathrm{LH}$ were impaired in their ability to determine temporal order. In contrast, Kagerer et al. (2002) and Harrington et al. (1998) both reported deficits in reproduction and estimation of stimulus duration in patients with right hemisphere lesions.

A very fruitful approach to studying auditory temporal information processing involves recording of the mismatch negativity (MMN) component of the auditory evoked-potential (AEP). The MMN is elicited automatically by deviations (e.g. pitch or duration changes) in an otherwise repetitive stream of auditory stimulation (De Sanctis et al., 2008; Näätänen, 1992; Ritter et al., 2002, 2006). It has been repeatedly shown that degree of temporal deviance and corresponding MMN amplitude correlate positively with each other (Amenedo and Escera, 2000; Desjardins et al., 1999; Kujala et al., 2002; but see Horváth et al., 2008). Furthermore, the presence or absence of an MMN response generally reflects behavioral performance, insofar as only deviants that can be discriminated at rates above chance are typically found to evoke a significant MMN response (e.g. Amenedo and Escera, 2000; Jaramillo et al., 2000). Uther et al. (2003) aimed to investigate hemispheric asymmetries in temporal resolution by delivering stimulus gaps monaurally to the left and right ear. In a gap-detection task ${ }^{1}$ the deviant (gap) was a 3, 5 , or $7 \mathrm{~ms}$ period of silence within an otherwise continuous auditory stimulus. As expected, Uther et al. (2003) found an enhanced MMN with increased gap size, but no differences in the laterality of the MMN. However, Uther et al. (2003) also found no evidence for the oft-replicated right ear advantage in gap-detection tasks (see Nicholls, 1996). Paavilainen et al. (1991) found an increased MMN response for duration changes over the right hemisphere independent of stimulated ear. That is, they found that the MMN response was larger over the right hemisphere for duration deviants presented to the left as well as to the right ear. Similar results were reported by Kasai et al. (2001) and Takegata et al. (2004) where source analysis of the duration induced MMN suggested right hemisphere predominance. Although the MMN tends to be larger over the right hemi-scalp for features other than duration (e.g. Paavilainen et al., 1991), the fact that it is also larger for duration deviants when duration is purportedly a function of left hemisphere auditory cortex would have to be considered rather puzzling. This is especially so when one considers that MMNs to language-specific stimuli show left-hemispheric dominance (e.g. Näätänen, 2001; Näätänen et al., 1997; Saint-Amour et al., 2007).

The primary purpose of the current study was to use the MMNsystem to interrogate right versus left hemisphere involvement in fine temporal discriminations. While previous MMN work has pointed to a greater contribution from the right hemisphere than would be expected if duration processing is indeed biased to the left hemisphere, none of these studies showing a right-hemisphere dominance

${ }^{1}$ In Uther et al. (2003) subjects performed separately a behavioral and electrophysiological experiment. In the latter case, subjects listened passively to the auditory stimulation. used near-threshold stimuli. The use of supra-threshold stimuli in these studies (i.e. duration differences that are easily detected) may have masked the essential contribution of the left hemisphere to fine timescale temporal differences. Here, we designed a study where we combined left/right ear stimulation to bias hemispheric processing with a manipulation of the degree of temporal deviation to estimate hemispheric asymmetries in discrimination accuracy at near-threshold temporal differences. A secondary goal of this study was to further examine a very interesting proposal by Opitz et al. (2002) that has posited an inverse relationship between activation of auditory cortical generators of the MMN within the temporal lobe and activation of putative frontal generators. They have contended that as the extent of deviance decreases such that deviants become less detectable and temporal-lobe generators of MMN decrease in responsivity, there is an increase in the activation of frontal generators, specifically within the inferior frontal gyrus (IFG; Opitz et al., 2002). The notion is that as deviance detection lessens in the superior temporal auditory regions, the frontal generator serves as a contrast-enhancement mechanism to amplify small changes (see Doeller et al., 2003). Both of these studies used frequency deviants but more recently, Rinne et al. (2005), using a duration-deviant, reported a similar increase in frontal activity for smaller deviants. In all above-mentioned studies (Doeller et al., 2003; Opitz et al., 2002; Rinne et al., 2005) the MMN-response during the electrophysiological recording was robust even for the smallest degree of deviance, with amplitudes reaching or exceeding $-2 \mu \mathrm{V}$ in value. Thus, even the smallest deviance used in these studies would have been clearly detectable for the participants, given the wealth of evidence relating MMN generation to perceptibility (e.g. Amenedo and Escera, 2000). In the present study we expressly manipulated the extent of deviance to ensure that participants were just capable of detecting the smallest deviants used (i.e. performance just above chance). By assessing the source strengths of equivalent current dipoles localized in superior temporal gyrus (STG) and IFG, we tested the notion of a contrastenhancement mechanism to amplify small threshold-level changes as posited by Opitz et al. (2002).

To presage our results, both the MMN and behavioural results confirm a prominent role for right hemisphere auditory cortices in early detection of small temporal duration deviations, showing that the right hemisphere is more sensitive to the smallest duration deviations we used than is the left hemisphere. The dipole source strength of the frontal MMN-generator did not modulate with the magnitude of the duration deviant. As such our data are not in line with the contrast-enhancement hypothesis suggested by Opitz et al. (2002).

\section{MATERIALS AND METHODS SUBJECTS}

Ten participants (five males) ranging in age from 18 to 36 years (mean 26.4) were included in the present study. All but 1 of the 10 participants were right handed as assessed using the Oldfield handedness inventory (Oldfield, 1971). The participants reported normal hearing and no known neurological deficits. Subjects received a modest fee for participating in the study. All provided written informed consent after the procedures were fully explained to them and the Internal Review Board (IRB) at the Nathan S. Kline Institute reviewed and approved all procedures. 


\section{STIMULUS AND PROCEDURE}

Participants viewed a silent film during stimulus delivery. Tones were presented monaurally via headphones (Sennheiser HD-600) at an intensity of $75 \mathrm{~dB}$ SPL in an acoustically and electrically shielded room. Standard stimuli ( $p=0.8$ ) were $440 \mathrm{~Hz}$ tones of $100 \mathrm{~ms}$ in duration and deviant stimuli $(p=0.2)$ were $115,130,145$ or $160 \mathrm{~ms}$, with a $10 \mathrm{~ms}$ rise and fall time. There were eight blocked conditions (four deviant types by two ears) where only one of the duration deviants was presented within a given block (i.e. only one ear was stimulated during each block). There were a total of 400 stimuli (i.e. 80 deviants) per block. The order of presentation of blocks was counterbalanced across participants. Each block-type was run four times per subject giving a total of 320 deviants per condition. Throughout each block, standard and deviant stimuli were delivered in a random manner with a stimulus onset asynchrony (SOA) of $750 \mathrm{~ms}$.

\section{EEG RECORDING AND ANALYSIS}

Brain activity was recorded and analyzed using the Neuroscan Synamp I system from 128 tin scalp electrodes (impedance $<5 \mathrm{k} \Omega$ ), referenced to the nose and digitized at $500 \mathrm{~Hz}$. The data were bandpass filtered from 1.5 to $45 \mathrm{~Hz}$ ( $24 \mathrm{~dB} /$ octave). Epochs of $500 \mathrm{~ms}$ were extracted with a $100 \mathrm{~ms}$ prestimulus baseline. Trials during which eye movements were made were rejected off-line on the basis of horizontal and vertical electro-oculogram. In addition, we applied an automatic artifact rejection criterion of $\pm 70 \mu \mathrm{V}$ for artifact rejection (e.g. blinks and large muscle activity), applied to all electrodes in the array. The average number of accepted trials per condition was 210 (ranging from 122 to 230).

\section{BEHAVIORAL DISCRIMINATION TASK}

A second cohort of 10 subjects (seven males) with an age-range from 22 to 33 years (mean 24.2) performed a behavioral discrimination task. The stimuli were precisely the same as in the electrophysiological recording sessions, but the SOA was lengthened by 150 to 900 ms to allow sufficient time for manual responses. A total number of 320 stimuli were presented per condition, 260 standard and 60 deviant tones in a single block. Subjects were instructed to press a button whenever they detected a deviant duration stimulus. There were eight blocked conditions (4 deviant types by two ears). The order of presentation of blocks was counterbalanced across participants.

\section{DATA ANALYSIS}

\section{Behavior}

In the behavioral task, the detection sensitivity measure of d-prime $\left(d^{\prime}\right)$ was calculated (Green and Swets, 1988). These $d^{\prime}$ values were subject to an analysis of variance (ANOVA) with the factors ear of delivery (left/right) and degree of temporal deviance (115, 130, 145 and $160 \mathrm{~ms}$ ).

\section{MMN-response}

Peak amplitude of MMN was measured in a $50 \mathrm{~ms}$ time window centered at the group-average peak latency at $\mathrm{Fz}$ for each condition. Subtracting the deviant-tone event-related potentials (ERPs) from the standard-tone ERPs delineated the MMN. A repeated measure ANOVA with factors ear of delivery and degree of temporal deviance $(115,130,145$ and $160 \mathrm{~ms})$ was conducted.

\section{Statistical cluster plots}

For exploratory purposes, pointwise two-tailed $t$-tests between standard-tone ERPs and deviant-tone ERPs were calculated at each time-point for all electrodes. The results of the pointwise $t$-tests from 128 electrodes are displayed as an intensity plot to efficiently summarize and facilitate identification of the onset and general topographic distribution of differential activation associated with standard-tone ERPs and deviant-tone ERPs. The $\mathrm{x}^{-}, \mathrm{y}-$, and $\mathrm{z}$ - axes, respectively, represents time, electrode location, and the $t$-test result (indicated by a color value) at each data point. This approach gives a statistical cluster plot (see Molholm et al., 2002; Murray et al., 2002) identifying both general scalp distribution and onset of differential responses between conditions across the entire epoch. We are aware that conclusions based on statistical cluster plots are undermined due to the large number of $t$-tests calculated across the electrode montage and recording epoch. In the present data treatment, periods of significant difference were only plotted if an alpha criterion of 0.05 or less was obtained and then only if this criterion was obtained for at least 11 consecutive data points (>22 ms at a $500 \mathrm{~Hz}$ digitization rate - see e.g. Foxe and Simpson, 2002; Guthrie and Buchwald, 1991; for similar approaches).

\section{Dipole source strength}

To investigate the change in dipole source strengths of the MMN component with increasing duration deviation, we performed source modeling using brain electric source analysis (BESA 5.1. software; Scherg and von Cramon, 1985; Simpson et al., 1995). Since we were not interested in hemispheric effects for this specific question, the MMN response over left and right ear presentation was averaged to increase the signal-to-noise term. BESA employs a least square fitting algorithm, defining location and orientation of dipoles for which the maximal amount of variance is explained (Scherg and Picton, 1991). For the propose of modeling, an idealized four-shell ellipsoidal head model with a radius of $90 \mathrm{~mm}$ and scalp and skull thickness of, respectively, 6 and $7 \mathrm{~mm}$ was assumed. Here, we fitted the orientation of a dipole configuration over a time window from 190 to $240 \mathrm{~ms}$, which spans the typical peak of the duration MMN component in our study. As for the dipole locations, we based our solution on several imaging studies which delineated common areas for duration MMN positioned in the superior temporal gyri and the right IFG (Dittmann-Balçar et al., 2001; Molholm et al., 2005; Rinne et al., 2005; Schall et al., 2003). For each dipole, source strength was calculated as the mean of the corresponding source waveform in a time window from 190 to $240 \mathrm{~ms}$. Dipole source strength was assessed for each subject and degree of duration deviance and analyzed using a one-way repeated-measures ANOVA.

\section{RESULTS \\ BEHAVIORAL}

The ANOVA of $d^{\prime}$ values in the behavioral task showed a significant main effect for degree of deviance $\left(F_{3,27}=125.1, p<0.001\right)$, and a significant ear of delivery by degree of deviation interaction $\left(F_{3,27}=4.49\right.$, $p=0.01)$. Follow-up paired $t$-tests revealed that d-prime for left and right ear presentation differed only for the $115 \mathrm{~ms}$ deviant $\left(t_{9}=-4.95\right.$, $p<0.001)$. Hence, interaction between ear of delivery and degree of deviation is mainly determined by higher d-prime values for $115 \mathrm{~ms}$ deviants delivered to the left ear (see Figure 1). 


\section{MMN RESPONSE}

The top panel of Figure 2A presents the grand mean ERPs averaged over 10 subjects elicited by the standard tone (black line) and the deviant tone presented to the left and right ear at the midline frontal
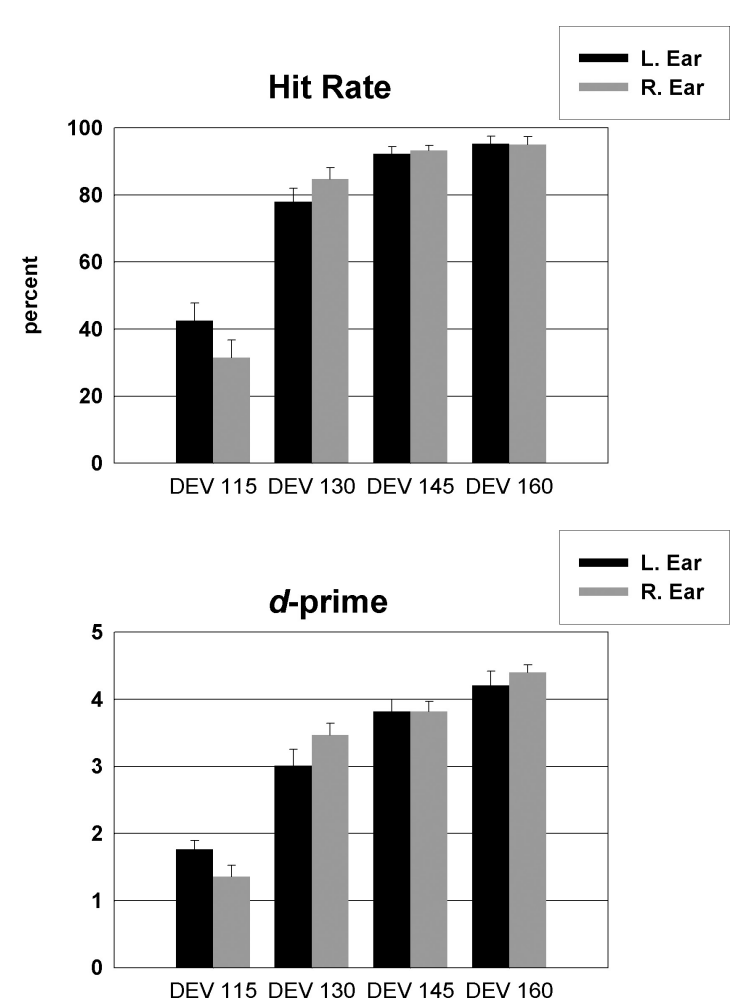

FIGURE 1 | Percent Hit-rates (top panel) and $d$-prime values (bottom panel) for each degree of temporal deviant and ear of delivery in the behavioral task. electrode site Fz. The bottom panel shows the subtracted waveform (deviant-tone ERP minus standard-tone ERP). For both left and right ear tone presentation there is a robust $\mathrm{MMN}$ response for the three greater deviance conditions with the 145 and 160 ms stimuli showing the largest MMN amplitude and the $130 \mathrm{~ms}$ stimuli showing a somewhat attenuated, but nonetheless highly robust, MMN. For the smallest degree of deviance (DEV 115) a MMN is only significant for tones presented to the left ear. Figure 2B shows the variance of the MMN responses represented by the standard errors for the $115 \mathrm{~ms}$ deviant tones presented to the left and right ear.

The ANOVA conducted on the MMN amplitude indicated a significant main effect for degree of deviance $\left(F_{3,27}=22.01, p<0.001\right)$ and an interaction between degree of deviance and ear of delivery $\left(F_{3,27}=4.6, p<0.05\right)$. Follow-up $t$-tests revealed that MMN amplitude for tones presented at the left and right side was significant $(p<0.05)$ in all conditions except for tones with the smallest degree of temporal deviance (i.e. DEV 115) delivered to the right ear (for DEV 115: $\left.t_{\mathrm{df}=9 \mid \text { left ear }}=-2.4, p=0.041 ; t_{\mathrm{df}=9 \mid \text { right ear }}=-1.2, p=0.24\right)$.

Scalp topographic maps representing interpolated potential distributions are shown in Figure 3. The MMN response at peak latency for each degree of deviance presented to left and right ear reveals a maximal focus over the right hemi-scalp. The only exception, where no lateralization but a more central distribution is evident can be seen for the smallest degree of deviance presented to the right ear. A maximal focus over the right hemi-scalp has been repeatedly reported as characteristic for the MMN response (e.g. Paavilainen et al., 1991), although not for speech stimuli (Saint-Amour et al., 2007).

Figure 4 presents a statistical cluster plot for the four levels of deviance. For the smallest degree of deviance, the cluster plot reveals significant differences over frontal cortex at $200 \mathrm{~ms}$ for deviants delivered to the left ear with a typical MMN distribution. In contrast, no differences are observed over fronto-central scalp during the typical MMN timeframe for the right ear. Rather, a later ( $290 \mathrm{~ms})$ cluster of significant difference is revealed over

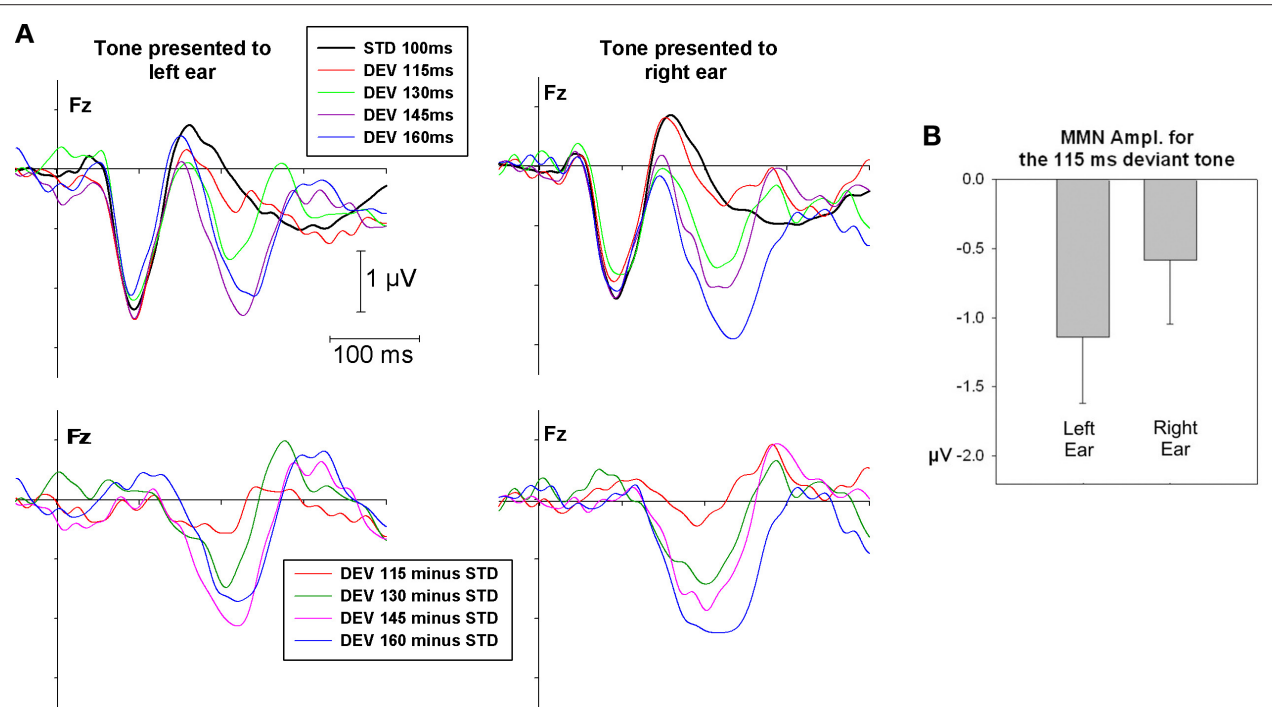

FIGURE 2 | (A) Grand mean ERPs at Fz for standard and deviant tones of 115, 130, 145 and 160 ms duration presented to the left and right ear (top panel) as well as MMN response (bottom panel) as subtraction waveform (deviant - standard). (B) Average MMN amplitude and standard error for the 115 ms deviant presented to the left and right ear. 


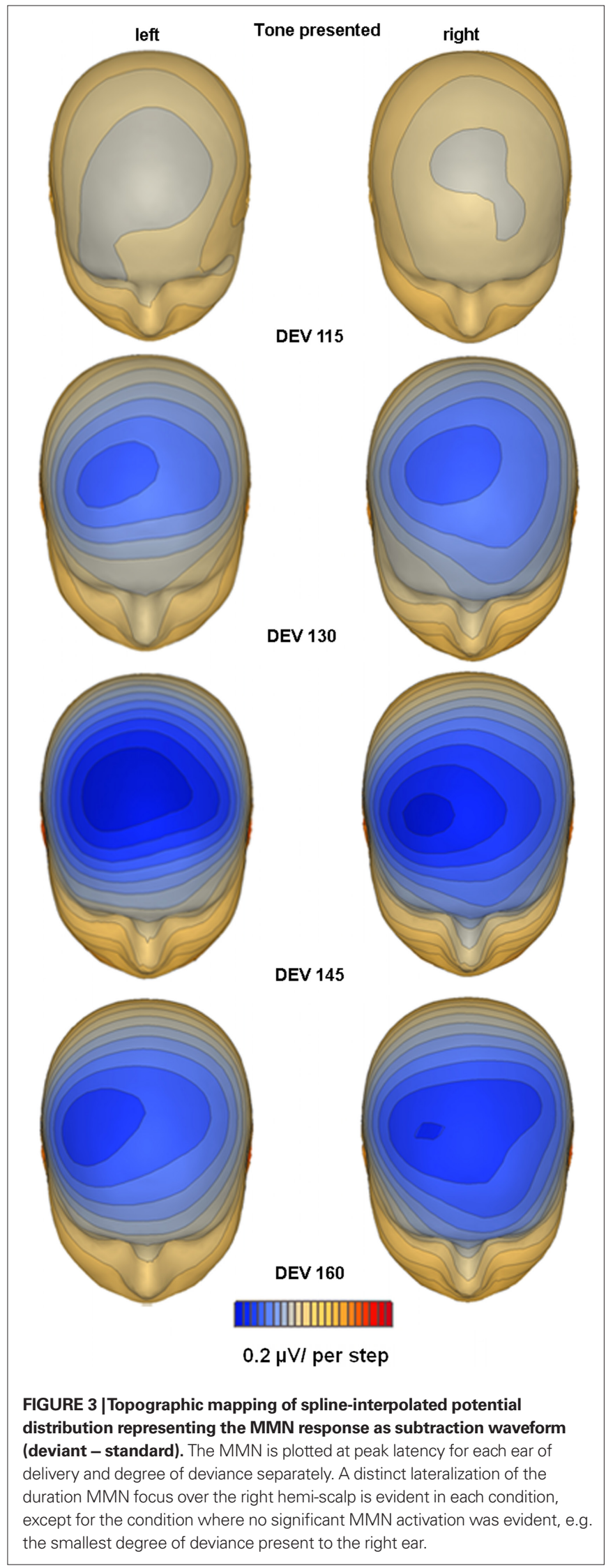

more posterior centro-parietal and parietal-occipital scalp. For the deviance of 130-160 ms presented to the left and right ear, we see an increasingly larger area of significant differences between standard and deviant AEPs during the typical MMN timeframe.

\section{DIPOLE SOURCE ANALYSIS}

Because our main question here was the effect of deviation on the strength of temporal and frontal dipole sources, we averaged ${ }^{2}$ the MMN response over left and right ear presentation. Figure 5 presents the source location and strength of MMN generators based on a 3-dipole solution. Dipoles were positioned in the left/right STG (Talairach coordinates: $-58,-23,9 / 58,-23,9)$ and right IFG $(46$, $20,6)$. Free fitting of dipole orientation across the modeled epoch (190-240 ms) resulted in a Goodness-of-fit of $>97 \%$.

To investigate the change in source strength of temporal and frontal dipoles determined by change in temporal deviation we calculated the mean of the dipole source waveforms in a time window from 190 to 240 ms. A repeated measurement ANOVA with factor degree of deviation was conducted separately for each dipole. Source waveform strength increased significantly with degree of deviation for the dipoles in the right $\left(F_{3,27}=26.2, p<0.000\right)$ and left superior temporal gyris $\left(F_{3,27}=18.9, p<0.000\right)$. In contrast, the source waveform for the right IFG dipole did not differ with degree of deviation $(p=0.36)$. In fact, the dipole fitted in the IFG showed essentially zero source-amplitude at the two lower levels of deviance.

\section{DISCUSSION}

In this study, measures of the MMN response and behavioral performance (d-prime) were employed to investigate fine temporal duration discrimination in the auditory modality. In particular, we sought to determine whether the left and right hemispheres differed in their discrimination sensitivity to near-threshold increments in stimulus duration. We also sought to further characterize the cortical generators of the duration MMN. Our specific focus was on determining if contributions from putative frontal MMN generators increased when the magnitude of deviation decreased, in line with the proposal of a frontal contrast-enhancement system, as posited by Opitz et al. (2002), Doeller et al. (2003) and Rinne et al. (2005).

The MMN has proven an excellent metric for assessing duration processing within the auditory cortex across a variety of tasks and stimulus configurations, such as gap detection (Desjardins et al., 1999), duration estimation (Amenedo and Escera, 2000) and interstimulus interval change (Kujala et al., 2001). The MMN paradigm is particularly well suited to these purposes for two main reasons. First, the neural mechanism underlying the processing of fine-grained temporal information can be assessed at an early pre-attentive level of information processing without invocation of higher-order cognitive processing as in a behavioral approach, where overt responding is required. Second, temporal deviations of as little as $5 \mathrm{~ms}$ have been shown to elicit the MMN (Desjardins et al., 1999). As such, the exquisite temporal resolution of the ERP approach allows us to investigate time discrimination mechanisms with high precision.

${ }^{2}$ For the smallest degree of temporal deviance ( $115 \mathrm{~ms}$ ) MMN response was source localized for left ear of presentation only, because there was no significant MMN response for $115 \mathrm{~ms}$ deviance presented to the right ear. 


\section{Left ear}
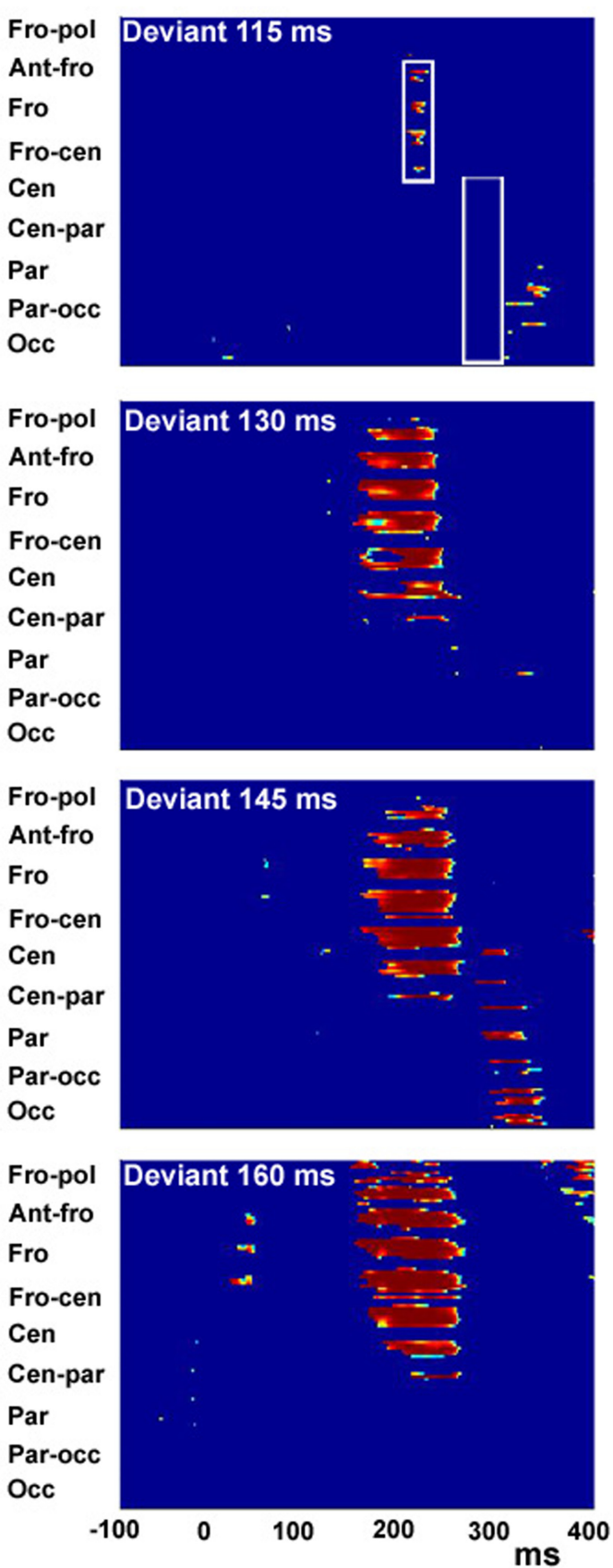

FIGURE 4 | Statistical cluster plots for degree of duration deviants of 115, 130, 145 and $160 \mathrm{~ms}$ presented to the left ear (left panel) and right ear (right panel). Color values indicate the result of pointwise $t$-tests evaluating differences between standard-tone and deviant-tone ERPs across time (x-axis)

\section{Right ear}
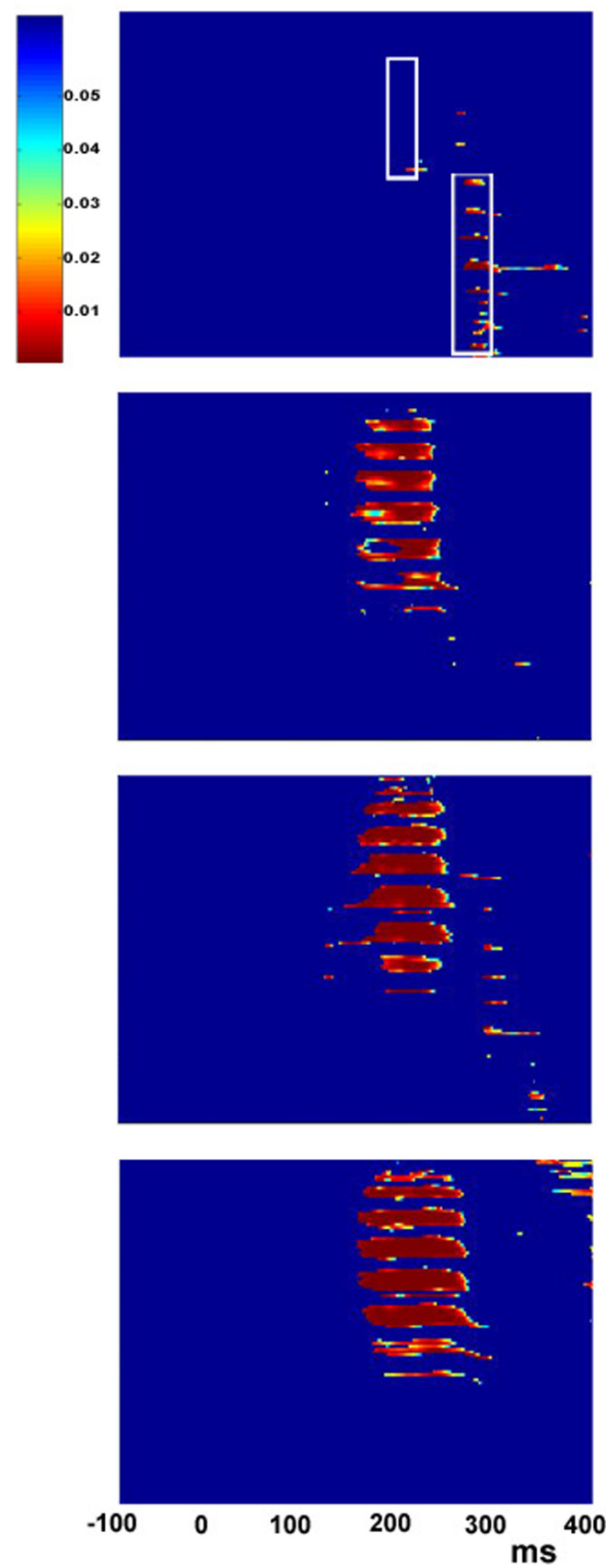

\section{HEMISPHERIC LATERALIZATION OF TEMPORAL PROCESSING}

To our knowledge, only Uther et al. (2003) have previously used the MMN paradigm to investigate left/right hemisphere differences in temporal processing. Uther et al. (2003) found no evidence for lateralization either at the pre-attentive level (MMN) or in behavioral measures, using a gap-detection task (e.g. 3, 5, or 7 ms periods of and electrode positions (y-axis) for the entire 128-electrode montage. For clarity, only p-values $<0.05$ are color encoded. Fro-pol, Fronto-polar; Ant-fro, Anteriorfrontal, Fro, Frontal; Fro-cen, Fronto-central; Cen, Central; Cen-par, Centralparietal; Par, Parietal; Par-occ, Parietal-occipital; Occ, Occipital. 


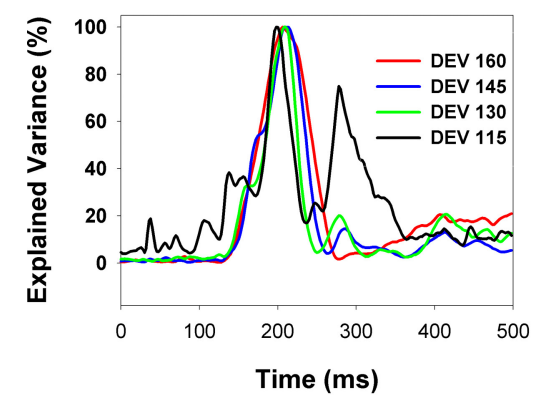

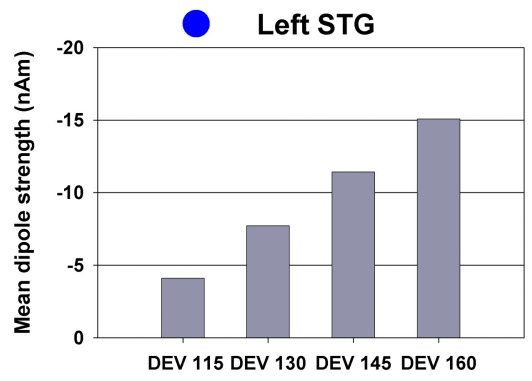

FIGURE 5 |The dipolar solution of the MMN component with sources bilateral in the superior temporal gyri and right inferior frontal gyrus. Dipoles are shown in the Talairach-transformed brain of an individual subject. Color dots by the graphs illustrating mean dipole strength indicate the corresponding color-
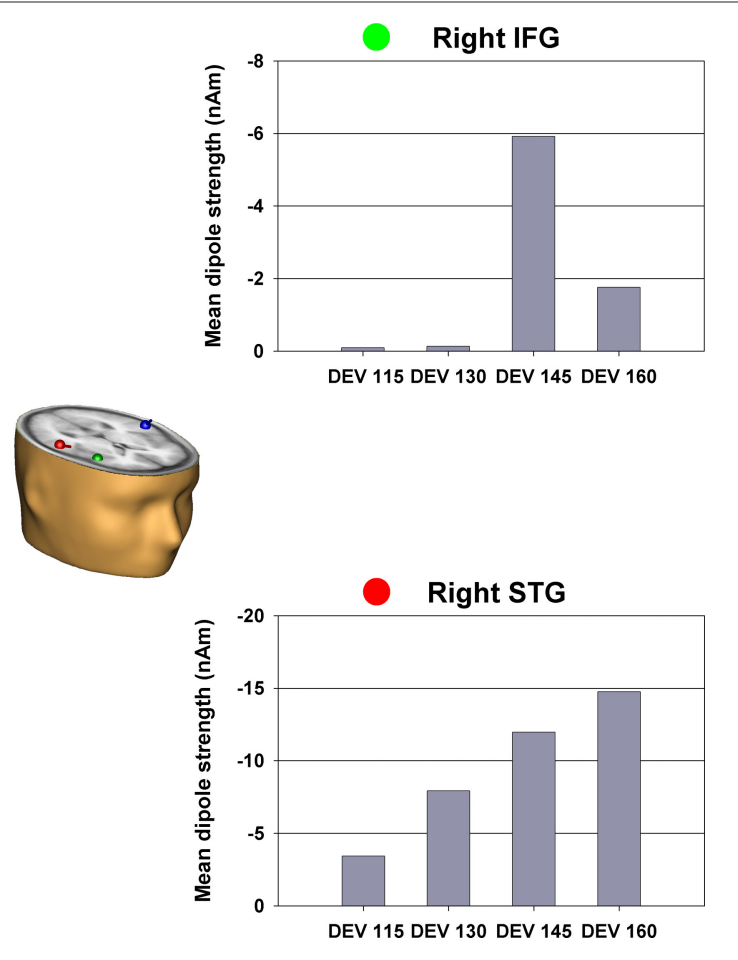

matched dipole. For each dipole and degree of deviation source strength was calculated as mean of the corresponding source waveform in a time window from 190 to $240 \mathrm{~ms}$. Mean strength of dipole in the left and right STG increased with degree of deviation. No significant change was observed for the right IFG dipole. for the smallest gap of 3 ms presented to the left or right ear, was over $87 \%$. Here we present evidence, both MMN and d-prime, indicating that the right hemisphere contribution to fine-grained temporal discrimination is just as strong, and indeed even somewhat stronger, as that of the left hemisphere. Interaction between left/right presentation and degree of deviation suggests the presence of a reliable MMN for the smallest degree of deviation, only when stimuli were presented to the left ear. Analysis of the high-density ERP visualised in the statistical cluster plot verified the presence of an MMN response over frontal and anterior-frontal areas for left ear deviants $(115 \mathrm{~ms}$ ) but not right ear deviants. In line with these electrophysiological findings, subsequent behavioural testing revealed significantly better temporal discrimination for the smallest duration deviants when they were presented to the left ear.

That we find a stronger RH contribution for duration judgments seems to conflict with the assumption of hemispheric asymmetry of fine temporal as well as language processing toward the LH. For example, Shannon et al. (1995) found that performance in speech recognition was remarkably high when preserving temporal but removing spectral cues of the speech signal, and a recent neuroimaging study found overlapping areas of activation for a gap-detection task and a syllable discrimination task, with these activations being stronger in left auditory cortex. (Zaehle et al., 2004).

The finding of a stronger $\mathrm{RH}$ contribution to duration discrimination here does however fall in line with several neuroimaging studies that similarly used duration judgment tasks to investigate lateralization of temporal processing (e.g. Belin et al., 2002; Harrington et al., 2004; Reiterer et al., 2005). In these studies, while a distributed bilateral network underlying duration judgment was observed, a positive correlation between performance level and fMRI/PET activation was only observed for areas within the right hemisphere. It is important to point out that in these three studies, where a stronger RH contribution was found, temporal judgments were based on stimulus durations/intervals that were equal to or $>100 \mathrm{~ms}$. This is germane because it has been suggested (Poeppel, 2003) that the absolute duration of signal segments that are used in a given experimental setting may have significant impact on which hemisphere is mainly recruited for their analysis.

A possible compromise position that could resolve the apparent left versus right hemisphere contradictions in the literature has come from the work of David Poeppel and his colleagues. Rather than a strict and simplistic dichotomization of functions between hemispheres, such as the rather extreme position that all temporal processing is achieved in the left hemisphere and all spectral processing in the right, he has posited a more complex division of labor between the hemispheres (Poeppel, 2003). The notion is that the auditory signal or scene must be analyzed on multiple different timescales and that this is achieved in non-primary auditory regions of both hemispheres, with each hemisphere having sensitivity to specific temporal structure in the signal. Based on results from psychophysical and neuropsychological data, Poeppel has argued that the left and right auditory cortices preferentially extract information on rapid $(<50 \mathrm{~ms})$ and slow $(>150 \mathrm{~ms})$ time 
scales respectively, which he has termed the "asymmetric sampling in time" (AST) model. A measure of support for the AST model has indeed been found in functional neuroimaging studies. For example, using sparse-sampling fMRI, Boemio et al. (2005) recorded the hemodynamic response to simple auditory signals constructed from repeating narrow-band noise segments that were varied in duration from fast (83 segments per second) to slow (three segments per second). This carefully conducted study controlled for all spectral confounds such that it was only along the temporal dimension that stimuli varied. In this way, they assessed the contributions of both hemispheres to temporal processing. The first important finding was that both left and right auditory cortices were highly sensitive to temporal (segmental) structure, primarily within the STG and superior temporal sulcus (STS). Equivalent sensitivity to local temporal structure was evident in the STG of both hemispheres. However, at the next level of processing in the STS, a marked hemispheric asymmetry emerged as a function of segment duration, with segment durations above 85 ms producing considerably stronger activation in the right dorsal STS. Similarly, a very recent study by Warrier et al. (2009) provided further support for this asymmetric sampling theory as they found stronger right lateralization for relatively slower rate stimuli $(3 \mathrm{~Hz})$ and leftward lateralization for fast rate stimuli $(33 \mathrm{~Hz})$. So, how well do the current results accord with the AST model? This is not entirely straightforward since the absolute segment durations we used were between 100 and $160 \mathrm{~ms}$ and so they were neither fast nor slow according to Poeppel's taxonomy, although they were weighted towards the slower range and as such should be expected to be favoured by the $\mathrm{RH}$. On the other hands, the MMN is not generated by the overall segment length but rather by the difference between the standard and deviant segments and these differences fall between 15 and $60 \mathrm{~ms}$, clearly within the theorized fast temporal sensitivity range of the LH.Also, it is with the $15 \mathrm{~ms}$ difference that we find the greatest asymmetry between $\mathrm{LH}$ and $\mathrm{RH}$ responsivity, in favour of the $\mathrm{RH}$. This latter result would appear to be in conflict with the AST model. Clearly more needs to be done to resolve this issue and a study that parametrically varied the segment length between fast and slower absolute values while using near-threshold duration deviants to generate the MMN might well provide a strong test of the AST.

Finally, it is also possible that the specific task employed to assess duration processing and discrimination may affect which hemisphere is shown to have greater involvement. In fact a preponderance of previous behavioral studies that have shown a LHA have used the so-called gap-detection task or a sequencing task (Nicholls, 1996). It is entirely plausible that the temporal processing necessary for gap-detection is not the same as that necessary for making a duration judgment, and that this accounts in part for the conflicting findings across studies. Nevertheless, our data clearly indicate a right hemisphere bias for fine-grained differential duration discriminations (of $15 \mathrm{~ms}$ here).

\section{THE FRONTAL CONTRAST-ENHANCEMENT THESIS}

As early as 1979, Näätänen proposed a frontal lobe contribution to the MMN based on two observations: (1) a more frontal distribution of the N1 for attended vs. unattended tones and (2) "high frontal amplitudes" for the MMN. However, the precise functional role of this frontal activity remains unresolved. Originally, this frontal contribution was conceived of as initiating an involuntary switch of attention to a sound change, after that change was first registered by the MMN detection mechanism in the STG (Näätänen, 1990; Näätänen and Michie, 1979). Subsequent studies have left little doubt that MMN leads to the involuntary reorienting of attention away from the attended stimulus, presumably toward the deviant stimulus (e.g., Escera et al., 2002; Schröger, 1996; Schröger and Wolff, 1998), though whether this is initiated by frontal generators in the timeframe of the MMN is not clear. Initial support for the contribution of a fronto/temporal network to the MMN came from the examination of MMN scalp current source density (CSD) topographies (Giard et al., 1990; and see also Deouell et al., 1998). Functional MRI studies using MMN paradigms have further supported a contribution from frontal areas, most often in the IFG (e.g. Doeller et al., 2003; Molholm et al., 2005). Of course, the usual caveat pertains to these functional imaging studies, in that they cannot determine whether these frontal contributions occur during the same timeframe as MMN generation or if they reflect later activation occurring after the MMN time-period has concluded.

Very little work has been done to directly test the original idea proposed by Näätänen and Michie (1979) that attributed the mechanism of involuntary attentional-switching to the frontal MMN-generator. In an attempt to distinguish the functional roles of the temporal and frontal MMN components Shalgi and Deouell (2007) manipulated sensory/perceptual and attentional factors and assessed their effects with the use of CSD. In accordance with Naatanen's and Giard's conceptualization of their respective roles, the sensory/perceptual factor was expected to affect temporal generators, whereas attentional manipulations were expected to affect frontal generators. Analysis of CSD topographic data suggested that only the sensory/perceptual factor affected the MMN, and that this was restricted to data from electrodes considered by the authors to reflect neuronal activity emanating from the temporal lobe, whereas modulation of the CSD MMN response from a set of electrodes considered to reflect activity emanating from frontal cortex was not seen. While caution must be taken in attributing activity from nearby sets of electrodes to completely separable neuronal generators, especially when sampling from fewer than 100 scalp sites as in Shalgi and Deouell (2007), there is no doubt that the data do not show compelling differences in activity over the more frontal regions as a function of condition. As such, these data are not in line with an attention-switching role for frontal activity in the timeframe of the MMN.

An alternative proposal is that frontal activity reflects a contrast gain mechanism that comes on line to facilitate detection of smaller differences. This was suggested by Opitz and colleagues (Doeller et al., 2003; Opitz et al., 2002) who, using fMRI and electrophysiology, found an inverse relationship between the right prefrontal gyrus (IFG) and the STG activation, with right IFG activation greater for small than medium and large pitch deviants. Rinne et al. (2005) replicated the fMRI results in terms of an inverse relationship using a duration deviant. As mentioned in the Section "Introduction," all three studies (Doeller et al., 2003; Opitz et al., 2002; Rinne et al., 2005) reported an increased hemodynamic IFG-response with a reduced degree of deviance. However, the MMN-response during the electrophysiological recording was robust even for the smallest degree of deviance used, reaching a very robust $-2 \mu \mathrm{V}$ in value, 
and in fact, Rinne et al. (2005) reported an MMN-response that did not differ for small, medium and large changes in duration. These MMN results suggest that even at the smallest degree of deviance used in these studies, these differences were clearly detectable, which raises some doubts about the functional role of the frontal MMN sources in terms of a contrast-enhancement mechanism for harder to detect deviations. We took advantage of the fact that our paradigm is well suited to addressing the hypothesis put forth by Opitz and colleagues and tested whether frontal contributions increased as deviance decreased. Our data however failed to support the contrast gain hypothesis. In fact, the dipole source-solution in the IFG showed essentially zero source-amplitude at the two lowest levels of deviance. As such our data are not in line with the notion of frontal sources serving as a contrast-enhancement mechanism in the timeframe of the MMN. Further research is required to explore the functional role of the IFG contribution during MMN-like paradigms. A distinct possibility is that these frontal activation uncovered with $\mathrm{fMRI}$, occur in a wholly different timeframe to those seen during MMN generation.

\section{ADDITIONAL FINDINGS}

The statistical cluster analysis for the $115 \mathrm{~ms}$ deviant condition in Figure 4 reveals two clusters of activation. A MMN activation over the frontal area at around $200 \mathrm{~ms}$ for stimuli presented to the left ear and a second cluster of activation over centro-parietal scalp at about $300 \mathrm{~ms}$ for stimuli presented to the right ear. Inspection of the ERP in Figure 2 indicates that the activation at about $300 \mathrm{~ms}$ is due to a positive-going deflection for deviant tones compared to standard tones when presented to the right ear. This positivity is present for many of the eight deviant conditions (see Figure 2, bottom panel) and resembles the $\mathrm{P} 3$ a component, which is generally thought to be associated with an automatic reorientation or shift of attention (e.g. Friedman et al., 2001). However, such an explanation for the $115 \mathrm{~ms}$ deviant condition does not fit with our performance data. In the behavioural testing, the $115 \mathrm{~ms}$ deviant stimuli were detectable at a much lower rate than deviants of 130-160 ms, which in our view renders it unlikely that the activation pattern at about $300 \mathrm{~ms}$ seen in the statistical cluster plot (Figure 4, top right panel) represents an automatic shift of attention. That is, it would be highly unusual for a P3a to be present in the right-115 ms condition where performance was extremely poor and virtually no MMN was elicited. It is also of note that the distribution suggested by the statistical cluster analysis suggests a response more posterior than would be expected for a P3a (Squires et al., 1975).

\section{ADDITIONAL CONSIDERATIONS}

A limitation of this study is that we did not collect behavioral and electrophysiological measures from the same group of subjects. Instead, our approach here was to keep our ERP subjects entirely naive to the purpose of the study, and they were simply instructed to ignore all auditory inputs. This is key during recording of the MMN because if subjects attend to the deviant stimuli or are asked to explicitly respond to them, then standard ERP novelty detection responses are evoked (i.e. the so-called N2/P3 complex - see Friedman et al., 2001). The N2 often overlaps in time with the MMN and this makes isolation and analysis of the MMN problematic (see Novak et al., 1990 for example). Nonetheless, had we collected a set of behavioral data from the ERP subjects after all recordings were made, this would potentially have allowed us to relate individual performance levels to MMN generation. We would reiterate though that the extant MMN literature already points to a very close relationship between the presence or absence of an MMN and the detectability of a given deviant (see e.g. Amenedo and Escera, 2000).

Another issue that needs to be considered pertains to the possibility that tones of different durations, even when presented at the same physical sound pressure level, can induce slight differences in perceived intensity due to the temporal integration of stimulus energy over short versus long periods. Might such perceived loudness differences have contributed to the current results? Todd and Mitchie (2000) addressed this issue of whether temporal integration of intensity might contribute to duration MMNs. In their study, they had two conditions, one in which intensity was adjusted and one in which it was not (for a $50 \mathrm{~ms}$ standard tone and a $125 \mathrm{~ms}$ duration deviant tone). Adjusting for perceived intensity did not affect the magnitude of the MMN at all, indicating that the MMN reflected the processing of duration deviance, and that the contribution of any perceived intensity differences was negligible. Further, for the most critical comparison made in the current study regarding hemispheric lateralization, the difference between standard and deviant tones was only $15 \mathrm{~ms}$, making it highly improbable that any perceived difference in loudness could be responsible for MMNs generated at this level of deviance.

\section{CONCLUSIONS}

We investigated the relative sensitivity of the left and right auditory cortex for temporal processing by recording the auditory MMN response to small duration deviants while stimuli were presented monaurally to either the left or right ear. Duration deviants differing by just $15 \%$ in duration relative to standard $100 \mathrm{~ms}$ tones elicited a significant MMN when stimuli were presented to the left ear (biasing the right hemisphere) but no detectable MMN was recorded for this same level of deviance when stimuli were presented to the right ear (biasing the left hemisphere). During these MMN recordings subjects ignored all stimuli. However, in a subsequent psychophysical study, participants detected significantly more of these $15 \%$ duration deviants and committed fewer false alarms for tones presented to the left ear, in close agreement with the MMN results. In contrast to the prevalent model, which posits greater temporal processing sensitivity for left hemisphere auditory cortex, these results point to equivalent if not greater right hemisphere contributions to temporal processing of small duration changes. In addition, we assessed a long-standing thesis that proposes a contrast-enhancement role for frontal cortical generators when deviance detection becomes more difficult and is not as effectively registered by the MMN system in temporal lobe auditory structures. Our data provide no support for this model.

\section{ACKNOWLEDGMENTS}

This work was supported in part by a grant from the US National Institute of Neurological Disorders and Stroke (NINDS RO1 NS30029) and from the US National Institute of Mental Health (NIMH RO1 MH65350). 


\section{REFERENCES}

Abramson, A.S. (1977). Laryngeal timing in consonant distinctions. Phonetica 34, 295-303.

Amenedo, E., and Escera, C. (2000). The accuracy of sound duration representation in the human brain determines the accuracy of behavioral perception. Eur. J. Neurosci. 12, 2570-2574.

Belin, P., McAdams, S., Thivard, L., Smith, B., Savel, S., Zilbovicius, M., Samson, S., and Samson, Y. (2002). The neuroanatomical substrate of sound duration discrimination. Neuropsychologia 40, 1956-1964.

Boemio A., Fromm, S., Braun, A., Poeppel, D. (2005). Hierarchical and asymmetric temporal sensitivity in human auditory cortices. Nat Neurosci. 8, 389-395.

Brown, C. P., Fitch, R. H., and Tallal, P. (1999). Sex and hemispheric differences for rapid auditory processing in normal adults. Laterality 4, 39-50.

Brown, S., and Nicholls, M. E. (1997). Hemispheric asymmetries for the temporal resolution of brief auditory stimuli. Percept. Psychophys. 59, 442-447.

Crow, T. J. (2006). March 27, 1827 and what happened later - the impact of psychiatry on evolutionary theory. Prog. Neuropsychopharmacol. Biol. Psychiatry 30, 785-796.

De Sanctis, P., Ritter, W., Molholm, S., Kelly, S. P., and Foxe, J. J. (2008). Auditory scene analysis: the interaction of stimulation rate and frequency separation on pre-attentive grouping. Eur. J. Neurosci. 27, 1271-1276.

Deouell, L.Y., Bentin, S., and Giard, M. H. (1998). Mismatch negativity in dichotic listening: evidence for interhemispheric differences and multiple generators. Psychophysiology 35, 355-365.

Desjardins, R. N., Trainor, L. J., Hevenor, S. J., and Polak, C. P. (1999). Using mismatch negativity to measure auditory temporal resolution thresholds. Neuroreport 10, 2079-2082.

Dittmann-Balçar, A., Jüptner, M., Jentzen, W., and Schall, U. (2001). Dorsolateral prefrontal cortex activation during automatic auditory duration-mismatch processing in humans: a positron emission tomography study. Neurosci. Lett. 308, 119-122.

Doeller, C. F., Opitz, B., Mecklinger, A., Krick, C., Reith, W., and Schröger, E. (2003). Prefrontal cortex involvement in preattentive auditory deviance detection: neuroimaging and electrophysiological evidence. Neuroimage 20, 1270-1282.

Escera, C., Corral, M. J., and Yago, E. (2002). An electrophysiological and behavioral investigation of involuntary attention towards auditory frequency, duration and intensity changes. Brain Res. Cogn. Brain Res. 14, 325-332.

Fitch, R. H., Brown, C. P., O’Connor, K. and Tallal, P. (1993). Functional lateralization for auditory temporal processing in male and female rats. Behav. Neurosci. 107, 844-850.

Foxe, J. J., McCourt, M. E., and Javitt, D. C. (2003). Right hemisphere control of visuospatial attention: line-bisection judgments evaluated with high-density electrical mapping and source analysis. Neuroimage 19, 710-726.

Foxe, J. J., and Simpson, G. V. (2002). Flow of activation from $\mathrm{V} 1$ to frontal cortex in humans. A framework for defining "early" visual processing. Exp. Brain Res. 142, 139-150.

Friedman, D., Cycowicz, Y. M., and Gaeta, H. (2001). The novelty P3: an event-related brain potential (ERP) sign of the brain's evaluation of novelty. Neurosci. Biobehav. Rev. 25, 355-373.

Gannon, P. J., Holloway, R. L., Broadfield, D. C., and Braun, A. R. (1998). Asymmetry of chimpanzee planum temporale: humanlike pattern of Wernicke's brain language area homolog. Science 279, 220-222.

Giard, M. H., Perrin, F., Pernier, J., and Bouchet, P. (1990). Brain generators implicated in the processing of auditory stimulus deviance: a topographic event-related potential study. Psychophysiology 27, 627-640.

Green, D.M., and Swets, J.A. (1988). Signal Detection Theory and Psychophysics. Los Altos, Peninsula Publishing.

Guthrie, D., and Buchwald, J. S. (1991). Significance testing of difference potentials. Psychophysiology 28, 240-244.

Harrington, D. L., Boyd, L. A., Mayer, A. R., Sheltraw, D. M., Lee, R. R., Huang, M., and Rao, S. M. (2004). Neural representation of interval encoding and decision making. Brain Res. Cogn. Brain Res. 21, 193-205.

Harrington, D. L., Haaland, K. Y., and Knight, R. T. (1998). Cortical networks underlying mechanisms of time perception. J. Neurosci. 18, 1085-1095.

Heilman, K. M., and Van Den Abell, T. (1980). Right hemisphere dominance for attention: the mechanism underlying hemispheric asymmetries of inattention (neglect). Neurology 30, 327-330.

Horváth, J., Czigler, I., Jacobsen, T., Maess, B., Schröger, E., and Winkler, I. (2008). MMN or no MMN: no magnitude of deviance effect on the MMN amplitude. Psychophysiology 45, 60-69.

Jaramillo, M., Paavilainen, P., and Näätänen, R. (2000). Mismatch negativity and behavioural discrimination in humans as a function of the magnitude of change in sound duration. Neurosci. Lett. 290, 101-104.

Kagerer, F. A., Wittmann, M., Szelag, E., and Steinbüchel, N. (2002). Cortical involvement in temporal reproduction: evidence for differential roles of the hemispheres. Neuropsychologia 40 357-366.

Kasai, K., Yamada, H., Kamio, S. Nakagome, K., Iwanami, A., Fukuda, M., Itoh, K., Koshida, I., Yumoto, M., Iramina, K., Kato, N., and Ueno, S. (2001). Brain lateralization for mismatch response to acrossand within-category change of vowels. Neuroreport 12, 2467-2471.

Kujala, A., Alho, K., Valle, S., Sivonen, P. Ilmoniemi, R. J., Alku, P., and Näätänen, R. (2002). Context modulates processing of speech sounds in the right auditory cortex of human subjects. Neurosci. Lett. 331, 91-94.

Kujala, T., Kallio, J., Tervaniemi, M., and Näätänen, R. (2001). The mismatch negativity as an index of temporal processing in audition. Clin. Neurophysiol. 112, 1712-1719.

Lackner, J. R., and Teuber, H. L. (1973) Alterations in auditory fusion thresholds after cerebral injury in man. Neuropsychologia 11, 409-415.

Leek, M. R., and Brandt, J. F. (1983). Lateralization of rapid auditory sequences. Neuropsychologia 21 , 67-77.

Molholm, S., Martinez, A., Ritter, W., Javitt, D. C., and Foxe, J. J. (2005). The neural circuitry of pre-attentive auditory change-detection: an fMRI study of pitch and duration mismatch negativity generators. Cereb. Cortex 15 545-551.

Molholm, S., Ritter, W., Murray, M. M., Javitt, D. C., Schroeder, C. E., and Foxe, J. J. (2002). Multisensory auditory-visual interactions during early sensory processing in humans: a highdensity electrical mapping study. Brain Res. Cogn. Brain Res. 14, 115-128.

Murray, M. M., Wylie, G. R., Higgins, B. A. Javitt, D. C., Schroeder, C. E., and Foxe, J. J. (2002). The spatiotemporal dynamics of illusory contour processing: combined high-density electrical mapping, source analysis, and functional magnetic resonance imaging. $J$. Neurosci. 22, 5055-5073.

Näätänen, R. (1990). The role of attention in auditory information processing as revealed by event-related potentials and other brain measures of cognitive function. Behav. Brain Sci. 13, 201-288.

Näätänen, R. (1992). Attention and Brain Function. Hillsdale, Erlbaum.

Näätänen, R. (2001). The perception of speech sounds by the human brain as reflected by the mismatch negativity (MMN) and its magnetic equivalent (MMNm). Psychophysiology 38, 1-21. Näätänen, R., Lehtokoski, A., Lennes, M., Cheour, M., Huotilainen, M., Iivonen, A., Vainio, M., Alku, P., Ilmoniemi, R. J., Luuk, A., Allik, J., Sinkkonen, J., and Alho, K. (1997). Language-specific phoneme representations revealed by electric and magnetic brain responses. Nature 385, 432-434.

Näätänen, R., and Michie, P. T. (1979). Early selective-attention effects on the evoked potential: a critical review and reinterpretation. Biol. Psychol. 8, 81-136.

Nicholls, M.E. (1996). Temporal processing asymmetries between the cerebral hemispheres: evidence and implications. Laterality 1, 97-138.

Novak, G. P., Ritter, W., Vaughan, H. G. Jr, and Wiznitzer, M. L. (1990) Differentiation of negative eventrelated potentials in an auditory discrimination task. Electroencephalogr. Clin. Neurophysiol. 75, 255-275.

Oldfield, R.C. (1971). The assessment and analysis of handedness: the Edinburgh inventory. Neuropsychologia 9, 97-113.

Opitz, B., Rinne, T., Mecklinger, A., von Cramon, D. Y., and Schroeger, E. (2002). Differential contribution of frontal and temporal cortices to auditory change detection: $\mathrm{AMRI}$ and ERP results. Neuroimage 15, 167-174

Paavilainen, P., Alho, K., Reinikainen, K., Sams, M., and Näätänen, R. (1991). Right hemisphere dominance of different mismatch negativities. Electroencephalogr. Clin. Neurophysiol. 78, 466-479.

Poeppel, D. (2003). The analysis of speech in different temporal integration windows: cerebral lateralization as "asymmetric sampling in time." Speech Commun. 41, 245-255.

Reiterer, S. M., Erb, M., Droll, C. D., Anders, S., Ethofer, T., Grodd, W., and Wildgruber, D. (2005). Impact of task difficulty on lateralization of pitch and duration discrimination. Neuroreport 16, 239-242.

Rinne, T., Degerman, A., and Alho, K. (2005). Superior temporal and inferior frontal cortices are activated by infrequent sound duration decrements: an fMRI study. Neuroimage 26, 66-72.

Ritter, W., De Sanctis, P., Molholm, S., Javitt, D. C., and Foxe, J. J. (2006). Preattentively grouped tones do not elicit MMN with respect to each other. Psychophysiology 43, 423-430.

Ritter, W., Sussman, E., Molholm, S., and Foxe, J. J. (2002). Memory reactivation or reinstatement and the 
mismatch negativity. Psychophysiology 39, 158-165.

Saint-Amour, D., De Sanctis, P., Molholm, S., Ritter, W., and Foxe, J. J. (2007). Seeing voices: high-density electrical mapping and source-analysis of the multisensory mismatch negativity evoked during the McGurk illusion. Neuropsychologia 45, 587-597 (Epub 2006 Jun 6).

Schall, U., Johnston, P., Todd, J., Ward, P. B., and Michie, P. T. (2003). Functional neuroanatomy of auditory mismatch processing: an event-related fMRI study of duration-deviant oddballs. Neuroimage 20, 729-736.

Scherg, M., and Picton, T. W. (1991). Separation and identification of event-related potential components by brain electric source analysis. Electroencephalogr. Clin. Neurophysiol. Suppl. 42, 24-37.

Scherg, M., and von Cramon, D. (1985). A new interpretation of the generators of BAEP waves I-V: results of a spatio-temporal dipole model. Electroencephalogr. Clin. Neurophysiol. 62, 290-299.

Schröger, E. (1996). A neural mechanism for involuntary attention shifts to changes in auditory stimulation. J. Cogn. Neurosci. 8, 527-539.

Schröger, E., and Wolff, C. (1998). Behavioral and electrophysiological effects of task-irrelevant sound change: a new distraction paradigm. Cogn. Brain. Res. 7, 71-87.

Shalgi, S., and Deouell, L. Y. (2007). Direct evidence for differential roles of temporal and frontal components of auditory change detection. Neuropsychologia 45, 1878-1888 (Epub 2007 Jan 18).

Shannon, R. V., Zeng, F. G., Kamath, V., Wygonski, J., and Ekelid, M. (1995). Speech recognition with primarily temporal cues. Science 270, 303-304.

Simpson, G.V., Foxe, J.J., Vaughan, H. G. Jr, Mehta, A. D., and Schroeder, C. E. (1995). Integration of electrophysiological source analyses, MRI and animal models in the study of visual processing and attention. Electroencephalogr. Clin. Neurophysiol. Suppl. 44, 76-92.

Squires, N. K., Squires, K. C., and Hillyard, S. A. (1975). Two varieties of long-latency positive waves evoked by unpredictable auditory stimuli in man. Electroencephalogr. Clin. Neurophysiol. $38,387-401$.

Steinbüchel, N., Wittmann, M., and Szelag, E. (1999). Temporal constraints of perceiving, generating, and integrating information: clinical indications. Restor. Neurol. Neurosci. 14, 167-182.

Sulakhe, N., Elias, L. J., and Lejbak, L. (2003). Hemispheric asymmetries for gap detection depend on noise type. Brain Cogn. 53, 372-375.
Takegata, R., Nakagawa, S., Tonoike, M., and Näätänen, R. (2004). Hemispheric processing of duration changes in speech and non-speech sounds. Neuroreport 15, 1683-1686.

Todd, J., Michie, P. T. (2000). Do perceived loudness cues contribute to duration mismatch negativity (MMN)? Neuroreport, 11, 3771-3774.

Uther, M., Jansen, D. H., Huotilainen, M., Ilmoniemi, R. J., and Näätänen, R. (2003). Mismatch negativity indexes auditory temporal resolution: evidence from event-related potential (ERP) and event-related field (ERF) recordings. Cogn. Brain Res. 17, 685-691.

von Steinbüchel, N., Wittmann, M., Strasburger, H., and Szelag, E. (1999). Auditory temporal-order judgment is impaired in patients with cortical lesions in posterior regions of the left hemisphere. Neurosci. Lett. 264, 168-171.

Vroon, P. A., Timmers, H., and Tempelaars, S. (1977). On the hemispheric representation of time. In Attention and Performance VI, S. Dornic ed. (Hillsdale, Lawrence Erlbaum Associates Inc.), pp. 231-245.

Warrier, C., Wong, P., Penhune, V. Zatorre, R., Parrish, T.,Abrams, D., and Kraus, N. (2009). Relating structure to function: Heschl's gyrus and acoustic processing. J. Neurosci. 29, 61-69.
Zaehle, T., Wüstenberg, T., Meyer,M., and Jäncke, L. (2004). Evidence for rapid auditory perception as the foundation of speech processing: a sparse temporal sampling fMRI study. Eur. J. Neurosci. 20, 2447-2456.

Conflict of Interest Statement: All of the authors attest that there are no conflicts of interest, financial or otherwise, pertaining to the work presented in this manuscript. Professor Foxe takes full responsibility for the integrity of the data and attests that all authors had full access to all the data in this study.

Received: 28 January 2009; paper pending published: 23 February 2009; accepted: 17 March 2009; published online: 20 April 2009

Citation: De Sanctis P, Molholm S, Shpaner $M$, Ritter W and Foxe JJ (2009) Right hemispheric contributions to fine auditory temporal discriminations: high-density electrical mapping of the duration mismatch negativity (MMN). Front. Integr. Neurosci. (2009) 3:5. doi: 10.3389/neuro.07.005.2009 Copyright (C) 2009 De Sanctis, Molholm, Shpaner, Ritter and Foxe. This is an openaccess article subject to an exclusive license agreement between the authors and the Frontiers Research Foundation, which permits unrestricted use, distribution, and reproduction in any medium, provided the original authors and source are credited. 